Noname manuscript No.

(will be inserted by the editor)

\title{
Recent progress on cluster and meron algorithms for strongly correlated systems
}

\author{
Debasish Banerjee
}

Received: date / Accepted: date

\begin{abstract}
Ab-initio studies of strongly interacting bosonic and fermionic systems is greatly facilitated by efficient Monte Carlo algorithms. This article emphasizes this requirement, and outlines the ideas behind the construction of the cluster algorithms and illustrates them via specific examples. Numerical studies of fermionic systems at finite densities and at real-times are sometimes hindered by the infamous sign problem, which is also discussed. The construction of meron cluster algorithms, which can solve certain sign problems are discussed. Cluster algorithms which can simulate certain pure Abelian gauge theories (realized as quantum link models) are also discussed.
\end{abstract}

Keywords Cluster Algorithms · Fermionic and Spin models · Lattice Gauge Theories

\section{Introduction: why cluster algorithms?}

The ab-initio studies of strongly correlated systems occurring in Nature, whether in particle physics or in condensed matter physics, is an extremely challenging topic. Analytical solutions are difficult to find in most cases, and perturbation theory fails for strong couplings. Certain weak coupling methods (such as the epsilon-expansion [1,2]) have been successful in addressing the existence of fixed points in renormalization group flow, as well as compute critical exponents at phase transitions by systematically improving over the mean field estimates. Large-N methods have provided another analytic handle on some interesting quantum field theories (QFTs) [3]. In the case of conformal field theories (CFTs) there has been exciting developments through the use of AdS/CFT [4, and the more recent conformal bootstrap [5] and the large charge

Saha Institute of Nuclear Physics, HBNI, 1/AF Bidhannagar, Kolkata 700064, India.

E-mail: debasish.banerjee@saha.ac.in 
expansion [6]. Tensor Networks [7] have significantly contributed to computing paradigms in lower dimensional systems, by opening up the possibility to simulate a large range of strongly interacting systems, even in real-time.

However, in the overwhelmingly large majority of cases, the Markov Chain Monte Carlo (MCMC) methods, starting from the initial proposal of Metropolis et al. [8] have provided an unbiased ab-initio method to numerically sample multidimensional integrals and evaluate the expectation values of physical operators. Let us denote the degrees of freedom in a (classical or quantum) system by $\left\{q_{i}\right\}, i=1, \cdots, V$, and the partition function for the system as

$$
\mathcal{Z}=\int \mathcal{D} q \mathrm{e}^{-S\left(\left\{q_{i}\right\}\right)}=\operatorname{Tr} \exp (-\beta H),
$$

where $S\left(\left\{q_{i}\right\}\right)$ is an action functional corresponding to the Hamiltonian $H$ at an inverse temperature $\beta$. The quantity $\mathrm{e}^{-S\left(\left\{q_{i}\right\}\right)}=W\left(\left\{q_{i}\right\}\right)$ is the Boltzmann weight and is typically positive for the chosen computational basis of $\left\{q_{i}\right\}$. If this is not the case, we encounter the sign problem and importance sampling fails. We will assume the positivity of the Boltzmann weight for the moment, and will come back to the exceptions later. The expectation value of a physical operator $\mathcal{O}$ (for example an order parameter or a correlation function) is

$$
\langle\mathcal{O}\rangle=\frac{1}{\mathcal{Z}} \int \mathcal{D} q \mathcal{O} \mathrm{e}^{-S\left(\left\{q_{i}\right\}\right)}
$$

Starting from an initial probability distribution, a Markov Chain is a series of probability distributions $\Pi_{k}\left(\left\{q_{i}\right\}\right)$, which steadily converge to the fixed point distribution $\Pi^{\star}\left(\left\{q_{i}\right\}\right)$, which is the equilibrium distribution $W\left(\left\{q_{i}\right\}\right)$. We will assume that the reader is familiar with the basics of Markov Chains, and local Monte Carlo algorithms and point to excellent textbooks which cover this topic in depth [9,10. While it is not always difficult to construct a Markov Chain with the necessary properties, the difficulty lies ensuring that the rate of convergence is insensitive to V. Further, even if a Markov chain were to converge to the equilibrium distribution, one needs to sample this distribution to obtain uncorrelated samples on which expectation values and correlation functions of relevant operators can be measured. This is exactly where improved algorithms, such as the cluster algorithm, assert their importance. To make this notion quantitative, we first introduce the concept of autocorrelation time.

Operationally, after equilibrium is reached, (local) Monte Carlo algorithm generates a set of configurations of $\left(\left\{q_{i}\right\}\right)$ according to the Boltzmann weight, on which physical operators are measured. Let us denote the distribution at the $l$-th Monte-Carlo time as $\left(\left\{q_{i}\right\}\right)_{l}$. Typically the distributions $\left(\left\{q_{i}\right\}\right)_{l}$ and $\left(\left\{q_{i}\right\}\right)_{l+1}$ are highly correlated since only a small change is involved in one step to the next. Therefore, measurement of the physical operator on these two subsequent configurations, $\mathcal{O}_{l}\left(\left\{q_{i}\right\}\right)$ and $\mathcal{O}_{l+1}\left(\left\{q_{i}\right\}\right)$ are also not independent.

Numerical calculations necessarily deal with finite data, let us denote this by $N_{\text {conf }}$. The sample mean of the dataset is $\overline{\mathcal{O}}=\frac{1}{N_{\text {conf }}} \sum_{l} \mathcal{O}_{l}$. According to the 
central limit theorem, the sample mean $\overline{\mathcal{O}}$ has a Gaussian distribution about the exact expectation value $\langle\mathcal{O}\rangle$, such that $\langle\mathcal{O}\rangle=\overline{\mathcal{O}} \pm \sigma_{\overline{\mathcal{O}}}$. With uncorrelated measurements, the best unbiased estimate of the error from the finite data-set is

$$
\sigma_{\overline{\mathcal{O}}}=\left[\frac{1}{N_{\mathrm{conf}}\left(N_{\mathrm{conf}}-1\right)} \sum_{k}\left(\mathcal{O}_{k}-\overline{\mathcal{O}}\right)^{2}\right]^{\frac{1}{2}}
$$

Note that we have used $\overline{\mathcal{O}}$ as the best estimate of the true mean, $\langle\mathcal{O}\rangle$. For large $N_{\text {conf }}$,

$$
\sigma_{\overline{\mathcal{O}}}^{2}=\left[\frac{1}{N_{\text {conf }}} \sum_{m}\left(\mathcal{O}_{m}-\overline{\mathcal{O}}\right) \frac{1}{N_{\text {conf }}} \sum_{n}\left(\mathcal{O}_{n}-\overline{\mathcal{O}}\right)\right]=\frac{1}{N_{\text {conf }}^{2}} \sum_{m, n}^{N_{\text {conf }}} \Gamma_{\mathcal{O}}(m-n) .
$$

The autocorrelation function, $\Gamma_{\mathcal{O}}(m)$, is a property of the Monte Carlo algorithm and is defined through the unequal time-correlator

$$
\Gamma_{\mathcal{O}}(m)=\left\langle\mathcal{O}_{m^{\prime}} \mathcal{O}_{m+m^{\prime}}\right\rangle-\langle\mathcal{O}\rangle^{2}=\Gamma_{\mathcal{O}}(-m) \stackrel{m \gg 1}{ } C \exp \left(-m / \tau_{\exp }\right)
$$

and decays exponentially at asymptotic times, with the decay time $\tau_{\text {exp }}$ corresponding to the slowest mode in the Monte Carlo dynamics. The integrated autocorrelation time, $\tau_{\text {int }}$, defined as

$$
2 \tau_{\mathrm{int}} \approx \sum_{m=-M}^{M} \Gamma_{\mathcal{O}}(m)=\Gamma_{\mathcal{O}}(0)+2 \sum_{m=1}^{M} \Gamma_{\mathcal{O}}(m)
$$

and the cut $M$ is due to finiteness of the dataset, and we have ignored corrections of order $\tau_{\exp } / N_{\text {conf }}$, for large $N_{\text {conf }}$. Note that $\Gamma_{\mathcal{O}}(0)$ is the sample variance. The expression for the error on the mean is then

$$
\sigma_{\mathcal{O}}^{2}=\frac{2 \tau_{\text {int }} \Gamma_{\mathcal{O}}(0)}{N_{\text {conf }}}=\frac{\Gamma_{\mathcal{O}}(0)}{N_{\text {eff }}}
$$

implying that there are only $N_{\text {eff }}$ independent configurations in the Markov chain. Unlike the equilibrium values, $\tau_{\text {int }}$ depends on the algorithm. Near a critical point, for example, autocorrelation times diverge with the physical correlation length $\xi$ as $\tau \sim c \xi^{z}$, and $z \sim 2$ for local algorithms ( $z$ is called the dynamical critical exponent). Such studies are therefore numerically challenging especially near the critical point, and the phenomena is called critical slowing down. Cluster algorithms can guarantee extremely small $\tau_{\text {int }}$, and sometimes even $z \sim 0$, thereby practically eliminating this problem.

\section{Quantum spin models}

Using the formulation of Fortuin and Kasteyln [11,12, the first cluster algorithm for simulating classical spin (the Ising and the Potts) models were constructed by Swendsen and Wang [13, and then extended by Niedermayer 
[14] and Wolff [15] for continuous spins systems. We refer to the review [16] for more details. Cluster algorithms for continuous systems, such as hard spheres, have also been developed. We do not discuss them here, but refer to [17 for a more detailed exposition. We instead focus on cluster algorithms for quantum systems, starting with quantum spins.

Cluster algorithms for quantum spin systems, in the world line formulation of quantum spins, were first developed in [18, 19, and extended to the continuous time version in 20. We note that the Stochastic Series Expansion (SSE) of the quantum spin Hamiltonian as developed in 21 leads to a loop Monte Carlo updating method [22, similar to the cluster algorithm. There has been significant developments in generalizing this algorithm for a variety of models, larger quantum spins, and we refer the reader to [23] for details and a more complete set of references. We note that the worm algorithm is another powerful idea which has led to the development of efficient algorithm for a large class of models, and in many cases is as powerful as the cluster algorithms. We refer to the interested reader to [24,35] for details.

Let us illustrate the construction of a cluster algorithm for the Heisenberg model, which not only has a very rich physical pedagogy behind it, but is also useful to understand the magnetic properties in certain electronic systems 25. This method is independent of spatial dimensions, but we will consider $(2+1)-\mathrm{d}$, anti-ferromagnetic version $J>0$ for definiteness. Starting from the Hamiltonian on a square lattice of linear extent $L$, the partition function $\mathcal{Z}$ at an inverse temperature $\beta$ is:

$$
\mathcal{Z}=\operatorname{Tr} \exp (-\beta H) ; \quad H=J \sum_{x, \hat{i}=1,2} \vec{S}_{x} \cdot \vec{S}_{x+\hat{i}} .
$$

Using the Suzuki-Trotter formula [26, we separate the Hamiltonian into 4 parts ( $2 d$ parts in $d$-spatial dimensions), $H=H_{1}+H_{2}+H_{3}+H_{4}$, such that the spins contained in each part mutually commute.

$$
\begin{aligned}
& H_{1}=J \sum_{x=(2 m, n)} \vec{S}_{x} \cdot \vec{S}_{x+\hat{1}} ; \quad H_{2}=J \sum_{x=(2 m+1, n)} \vec{S}_{x} \cdot \vec{S}_{x+\hat{1}} ; \\
& H_{3}=J \sum_{x=(m, 2 n)} \vec{S}_{x} \cdot \vec{S}_{x+\hat{2}} ; \quad H_{4}=J \sum_{x=(m, 2 n+1)} \vec{S}_{x} \cdot \vec{S}_{x+\hat{2}} .
\end{aligned}
$$

We construct $\mathcal{Z}$ by inserting intermediate time-slices, such that $\beta=4 N \epsilon$, and $\epsilon$ is the temporal lattice spacing. Using $\mathcal{I}=\left|n_{k}\right\rangle\left\langle n_{k}\right|$, we expand $\mathcal{Z}$ as a product over the matrix elements of the transfer matrix, expressed in the $S^{z}= \pm \frac{1}{2}$ basis, which is chosen as the computational basis:

$$
\begin{aligned}
\mathcal{Z} & =\operatorname{Tr} \exp (-\beta H)=\lim _{\substack{N \rightarrow \infty \\
\epsilon \rightarrow 0}} \sum_{n}\left\langle n\left|\left[\mathrm{e}^{-\epsilon\left(H_{1}+H_{2}+H_{3}+H_{4}\right)}\right]^{N}\right| n\right\rangle \\
& =\lim _{\substack{N \rightarrow \infty \\
\epsilon \rightarrow 0}} \sum_{\substack{\{n\} \\
n_{4 N}=n_{0}}} \prod_{k=0}^{N-1}\left\langle n_{k}\left|\mathrm{e}^{-\epsilon H_{1}}\right| n_{k+1}\right\rangle\left\langle n_{k+1}\left|\mathrm{e}^{-\epsilon H_{2}}\right| n_{k+2}\right\rangle\left\langle n_{k+2}\left|\mathrm{e}^{-\epsilon H_{3}}\right| n_{k+3}\right\rangle\left\langle n_{k+3}\left|\mathrm{e}^{-\epsilon H_{4}}\right| n_{k+4}\right\rangle
\end{aligned}
$$


We have used the Trotter formula in the second line, and the matrix elements connect only the specific bonds. Finally, we can express the matrix elements via the local action

$$
\mathcal{Z}=\prod_{x, t} \sum_{s(x, t)= \pm 1} \mathrm{e}^{-S\left[s_{1}, s_{2}, s_{3}, s_{4}\right]}
$$

where the term $S\left[s_{1}, s_{2}, s_{3}, s_{4}\right]$ connects two neighboring spins, $s_{1}, s_{2}$ at a timeslice, $t$, with their forward-in-time partners $s_{3}, s_{4}$. Note that only a set of bonds are active at a given time-slice, and all others are passive, and this 4-spin interaction traces an active plaquette. A schematic figure for the Trotter decomposition in $(1+1)-\mathrm{d}$ is shown in Fig 1 (left), where the shaded plaquettes are the active ones, and indicate the spin pairs which are interacting at that given time. A representative $s_{1}, s_{2}, s_{3}, s_{4}$ are also shown in the same figure. For the anti-ferromagnetic Heisenberg model, the explicit values of the transfer matrix (in the basis where the states of the two spins at sites $x$ and $y$ are $|\uparrow \uparrow\rangle,|\uparrow \downarrow\rangle,|\downarrow \uparrow\rangle,|\downarrow \downarrow\rangle)$ are:

$$
\mathrm{e}^{-S\left[s_{1}, s_{2}, s_{3}, s_{4}\right]}=\left\langle s_{1} s_{2}\left|\mathrm{e}^{-\epsilon J \vec{S}_{x} \vec{S}_{y}}\right| s_{3} s_{4}\right\rangle=\mathrm{e}^{-\frac{\epsilon J}{4}}\left[\begin{array}{cccc}
1 & 0 & 0 & 0 \\
0 & \frac{1}{2}\left(1+\mathrm{e}^{\epsilon J}\right) & \frac{1}{2}\left(1-\mathrm{e}^{\epsilon J}\right) & 0 \\
0 & \frac{1}{2}\left(1-\mathrm{e}^{\epsilon J}\right) & \frac{1}{2}\left(1+\mathrm{e}^{\epsilon J}\right) & 0 \\
0 & 0 & 0 & 1
\end{array}\right]
$$

Note that there are only two off-diagonal elements, both of which are negative. For a bipartite lattice, the negative sign can be eliminated by doing a unitary transformation on every alternative spin, such that the off-diagonal elements become $\frac{1}{2}\left(\mathrm{e}^{\epsilon J}-1\right)$. For a triangular lattice, for example (or for any non-bipartite lattice, in general), this does not work, and we encounter the first example of a sign problem. In the chosen basis the probability weights are not positive definite, and Monte Carlo simulation cannot be performed.

Next, to construct a cluster algorithm we have to expand the configuration space by including bond variables, together with the quantum spins. This implies choosing the bond variables $[b]$ such that the following equation is satisfied for the different possibilities of $s_{1}, s_{2}, s_{3}, s_{4}$,

$$
\mathrm{e}^{-S\left[s_{1}, s_{2}, s_{3}, s_{4}\right]}=\sum_{[b=A, B]} \mathrm{e}^{-S\left[s_{1}, s_{2}, s_{3}, s_{4} ; b\right]}
$$

In this particular example, as shown in Fig 1, the linear equations can be satisfied by the use of two bond breakups, $A$ and $B$. The clusters are constructed as follows: (a) start from an initial site, (b) follow the chosen breakups as explained in the caption of Fig 1 (right) until the initial point is reached. In this case, the resulting cluster is the same as a loop, and hence the relation to the loop algorithm. Flipping the cluster implies the operation $\left|\uparrow_{k}\right\rangle \leftrightarrow\left|\downarrow_{k}\right\rangle$, which preserves the Boltzmann weight of the configuration, and yet changes the configuration globally, thereby decorrelating them very fast. This is the reason for the efficiency of the cluster algorithm - the clusters correspond to 


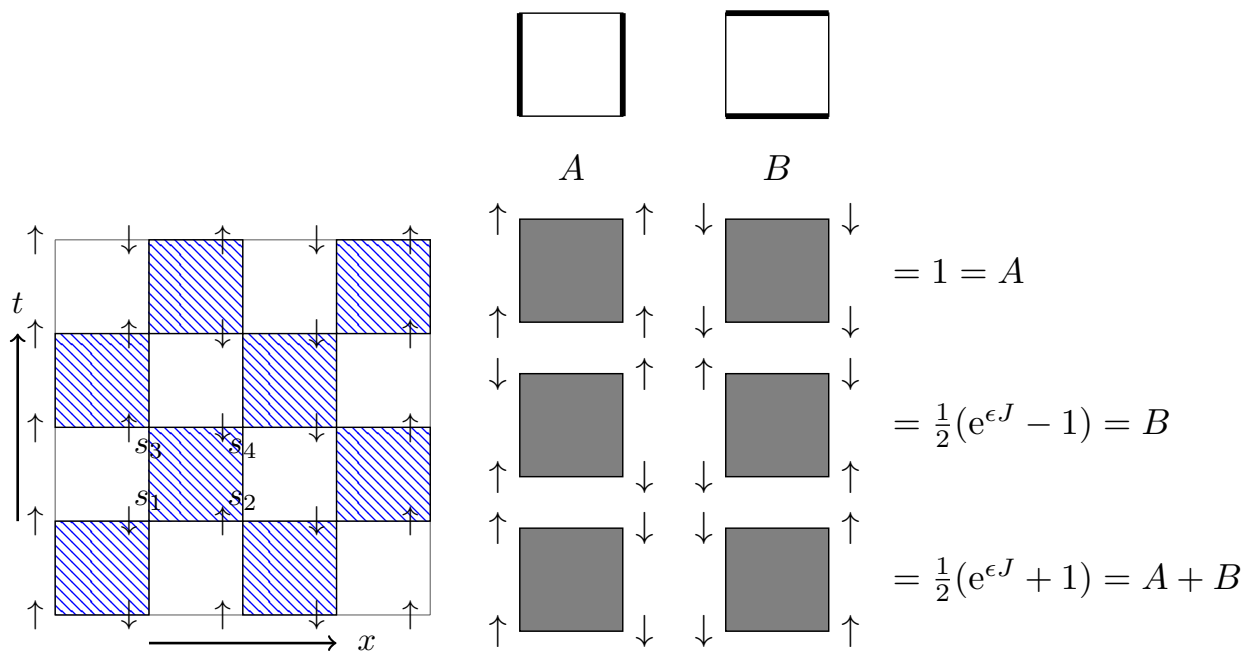

Fig. 1 (left) An example of a world-line configuration of the anti-ferromagnetic Heisenberg model. There is one transition and one anti-transition which spatially displaces a spinup (down) and brings it back. (right) Possible bond-types for this model: (a) the A-type breakup connects identical spins forward in time, (b) the B-type breakup connects opposite (spin-up with spin-down) spins sideways. We can figure out the probability of the weights by computing which breakup can be applied for which interaction plaquette. For those where the spin-states do not, and cannot change (top), only A can be applied, whereas where the spin state flips (middle) only B can be applied. The last case is where the spin state can change but did not. Here, the A-breakup can be applied with the probability $W_{A}=A /(A+B)=2 /(\exp (\epsilon J)+1)$.

correlated degrees of freedom, which get efficiently updated by flips. In fact, representing the configurations in terms of clusters allows one to construct improved estimators for quantities such as the magnetization, susceptibility and the correlation function.

Let us illustrate the construction of improved estimators through examples. The total (uniform) magnetization of the spins is given as $\mathcal{M}\left[\left\{S^{z}\right\}\right]=$ $\sum_{i}\left(S^{z}\right)_{i}=\sum_{C} \mathcal{M}_{C}$, where $i$ runs over all the space-time lattice sites, and $C$ runs over all the clusters into which a single configuration of spins has been decomposed. The equality follows since each spin must uniquely belong to a cluster. The cluster magnetization is thus given as $\mathcal{M}_{C}=\sum_{i \in C}\left(S^{z}\right)_{i}$. Since each cluster flip changes the sign of $\mathcal{M}_{C}$, we conclude that $\langle\mathcal{M}\rangle=0$. Note that this is consistent with the physical result that the magnetization always vanishes in a finite volume. Such results are extremely difficult to observe, especially in the broken phase, with local update algorithms. Another example 
is the (uniform) susceptibility:

$$
\chi=\frac{\beta}{V}\left\langle\mathcal{M}^{2}\right\rangle=\frac{\beta}{V}\left\langle\left(\sum_{C} \mathcal{M}_{C}\right)^{2}\right\rangle=\frac{\beta}{V}\left\langle\sum_{C_{i}, C_{j}} \mathcal{M}_{C_{i}} \mathcal{M}_{C_{j}}\right\rangle=\frac{\beta}{V}\left\langle\sum_{C} \mathcal{M}_{C}^{2}\right\rangle
$$

Note that different clusters $C_{i}$ and $C_{j}$ are uncorrelated and hence the crossterms vanish under the operation of all cluster flips, leaving behind only diagonal terms. Further in a cluster $C$, since all the spins are pointing in the same direction, the magnetization is given by the total cluster size, $\mathcal{M}_{C}= \pm|C|$. This allows us to rewrite $\chi$ as follows, $\chi=\frac{\beta}{V}\left\langle\sum_{C}|C|^{2}\right\rangle$. Thus it is evident that clusters are relevant physical objects, since their size is related to a physical quantity.

Two variants of this cluster algorithm are well-known: multi-cluster algorithm, which proceeds to construct all the possible clusters on a given configuration of spins and bonds, and then flips each cluster with a $50 \%$ probability, and the single-cluster, or the Wolff algorithm which constructs a single cluster at always flips it. It is also possible to construct this cluster algorithm in continuous time.

It is to be noted that both types of cluster algorithms suffer from Trotter errors, caused due to the finite $\epsilon$ essential in the numerical simulations. In particular, the errors on the physical quantities go as $\epsilon^{2}$, and thus one typically simulates for several different $\epsilon$ values to take the time-continuum limit. It is however possible to construct the cluster algorithms directly in the timecontinuum limit [20], which eliminate the Trotter errors completely.

It must be emphasized that for the cluster algorithm to be successful, certain special configurations called reference configurations need to exist. In the case of the Heisenberg anti-ferromagnet the reference configuration(s) are the ones where the spins are staggered on the bi-partite lattice. Any configuration can then be decomposed into a certain set of clusters, and the clusters are accordingly flipped to bring the configuration into (one of) the reference configurations. This trivially proves ergodicity. In many cases (examples are frustrated magnets, spin glasses, gauge theories), while it is conceivable to build clusters, absence of reference configurations causes the clusters to grow too big, almost filling up the whole volume. In such a case, the cluster algorithm does not perform any better than a local update algorithm.

\section{Fermions}

Attempting to simulate fermions brings us head on with the fermion sign problem. This is most directly seen in the world-line representation of the fermions in the occupation number basis. In dimensions $d \geq 2$, it is easy to construct world-lines of identical fermions which twist around each other as they evolve in Euclidean time. Configurations which have fermions exchanging positions an odd number of times have an overall negative sign compared with those where fermions exchange positions an even number of times (everything 
else being identical), due to the Pauli exclusion principle. A particularly clear statement of this fermion sign problem and what it entails to solve the problem is given in 27 .

As in the case of quantum spins, to simulate fermionic systems we construct the partition function as usual. In the Lagrangian formulation, one uses Grassmann variables, which is typically integrated out to leave behind a determinant involving auxiliary fields (if the theory has four-Fermi interactions, or Yukawa couplings), or even gauge fields (as in quantum electrodynamics, or quantum chromodynamics). Updating procedures either involve updating the determinant directly 28, 29], or recast the determinant in terms of bosonic fields, which are then updated 30 . To construct cluster algorithms for fermions, we first obtain a bosonic representation using the Jordan-Wigner transformation. If the resulting bosonic system can be efficiently updated using a cluster algorithm, then one can identify types of interactions for which the fermion sign problem can be solved [31. When such an approach succeeds, it is known as the meron algorithm first introduced in [32, and will be discussed below. Other novel approaches, such as the fermion bag [33, 34] or the diagrammatic Monte Carlo [35, 36] are getting increasingly popular in simulating fermionic systems. The former method expands the fermionic action and groups regions where the fermions can propagate freely against regions where they are bound into monomers and dimers. The Monte Carlo procedure then updates these regions, which are denoted as fermion bags. The latter approach directly samples the Feynman diagrams associated with the interactions, and in certain regimes can be related to the bag approach.

Before discussing the construction of the meron algorithm, we elaborate the nature of the fermionic sign problem, which will also serve to understand the solution. First, we set up the fermionic path integral in the occupation number basis $\left\{n_{k}\right\}$ :

$$
\mathcal{Z}_{\mathrm{f}}=\operatorname{Tr} \exp \left(-\beta H_{\mathrm{f}}\right)=\sum_{\left\{n_{k}\right\}} p\left(\left\{n_{k}\right\}\right) ;\langle A\rangle=\frac{1}{\mathcal{Z}_{\mathrm{f}}} \sum_{\left\{n_{k}\right\}} A\left(\left\{n_{k}\right\}\right) p\left(\left\{n_{k}\right\}\right),
$$

where $p\left(\left\{n_{k}\right\}\right)$ is the probability for a given configuration, and $A$ is an operator in the occupation number basis. To qualify the severity of this problem, one can consider the sign of the configuration as a part of the observable $A$ through the decomposition $p\left(\left\{n_{k}\right\}\right)=\operatorname{sign}\left(\left\{n_{k}\right\}\right)\left|p\left(\left\{n_{k}\right\}\right)\right|$, the sign being \pm 1 depending on whether the fermions in the configuration swap their positions even or odd number of times. It follows that

$$
\begin{aligned}
\langle A\rangle_{\mathrm{f}} & =\frac{\sum_{\left\{n_{k}\right\}} A\left(\left\{n_{k}\right\}\right) p\left(\left\{n_{k}\right\}\right)}{\sum_{\left\{n_{k}\right\}} p\left(\left\{n_{k}\right\}\right)} \\
& =\frac{\sum_{\left\{n_{k}\right\}} A\left(\left\{n_{k}\right\}\right) \operatorname{sign}\left(\left\{n_{k}\right\}\right)\left|p\left(\left\{n_{k}\right\}\right)\right|}{\sum_{\left\{n_{k}\right\}}\left|p\left(\left\{n_{k}\right\}\right)\right|} \times \frac{\sum_{\left\{n_{k}\right\}}\left|p\left(\left\{n_{k}\right\}\right)\right|}{\sum_{\left\{n_{k}\right\}} \operatorname{sign}\left(\left\{n_{k}\right\}\right)\left|p\left(\left\{n_{k}\right\}\right)\right|} \\
& =\frac{\langle A \cdot \operatorname{sign}\rangle_{\mathrm{b}}}{\langle\operatorname{sign}\rangle_{\mathrm{b}}}
\end{aligned}
$$


The subscript $\mathbf{b}$ indicates that the averaging is done over a bosonic system whose weights, $\mid p\left(\left\{n_{k}\right\} \mid\right.$, are positive definite by construction. The quantity $\langle\operatorname{sign}\rangle_{\mathrm{b}}$ measures the severity of the sign problem. It can shown that $\langle\operatorname{sign}\rangle_{\mathrm{b}}=$ $\exp (-\beta V \Delta f)$, where $\Delta f=f_{\mathrm{f}}-f_{\mathrm{b}}$ is the difference in free energy density between the fermion and bosonic ensembles. At low temperatures and large volumes, this quantity is exponentially small:

$$
\frac{\left(\sigma_{\mathrm{sign}}\right)_{\mathrm{b}}}{\langle\operatorname{sign}\rangle_{\mathrm{b}}}=\frac{\left[\left\langle\operatorname{sign}^{2}\right\rangle_{\mathrm{b}}-\langle\operatorname{sign}\rangle_{\mathrm{b}}^{2}\right]^{\frac{1}{2}}}{\sqrt{N}\langle\operatorname{sign}\rangle_{\mathrm{b}}} \approx \frac{\exp (\beta V \Delta f)}{\sqrt{N}},
$$

where $N$ is the number of uncorrelated data, and we have used $\left\langle\operatorname{sign}^{2}\right\rangle_{\mathrm{b}}=$ $1,\langle\operatorname{sign}\rangle_{\mathrm{b}} \approx 0$. Clearly, $N \sim \exp (2 \beta V \Delta f)$ to determine the sign. Thus even if $\langle A\rangle_{\mathrm{f}} \sim 1$, it is measured as the ratio of two exponentially small signals, and requires exponential effort to extract from statistical noise.

One could aim to cancel the negative signs by pairing the configurations which carry an -1 sign with those that carry a 1 sign, such that only a few unmatched configurations remain. In fact, this is the idea behind the meron algorithm. It is however important to realize that this solves only one-half of the sign problem. With the matching, we effectively have $\operatorname{sign}^{2}=\operatorname{sign}$, since sign $=0,1$ and hence

$$
\frac{\left(\sigma_{\text {sign }}\right)_{\mathrm{b}}}{\langle\operatorname{sign}\rangle_{\mathrm{b}}}=\frac{\left[\langle\operatorname{sign}\rangle_{\mathrm{b}}-\langle\operatorname{sign}\rangle_{\mathrm{b}}^{2}\right]^{\frac{1}{2}}}{\sqrt{N}\langle\operatorname{sign}\rangle_{\mathrm{b}}} \approx \frac{\exp (\beta V \Delta f / 2)}{\sqrt{N}},
$$

Note that we have achieved an exponential gain in statistics, but one still needs $N \sim \exp (\beta V \Delta f)$ for any meaningful result. The meron algorithm incorporates the two steps together: it identifies the configurations which can be analytically cancelled, and never generates them. Thus, one always generates configurations which contribute non-trivially to $\mathcal{Z}_{\mathrm{f}}$. The algorithm, however, only works for a restricted class of interactions.

Let us illustrate this for the $\mathrm{t}-\mathrm{V}$ model in $(2+1)-\mathrm{d}$, extensively used in the study of certain electronic systems (though the algorithm can be constructed in any dimensions). The Hamiltonian is

$$
\mathrm{H}_{\mathrm{f}}=-\frac{\mathrm{t}}{2} \sum_{x, \hat{i}=1,2}\left(c_{x}^{\dagger} c_{x+\hat{i}}+c_{x+\hat{i}}^{\dagger} c_{x}\right)+\mathrm{V} \sum_{x, \hat{i}=1,2}\left(n_{x}-\frac{1}{2}\right)\left(n_{x+\hat{i}}-\frac{1}{2}\right),
$$

with $V \geq t>0$. The fermion creation (annhiliation) operators at site $\mathrm{x}$, $c_{x}\left(c_{x}^{\dagger}\right)$ satisfy the following anti-commutation relations: $\left\{c_{x}, c_{y}\right\}=\left\{c_{x}^{\dagger}, c_{y}^{\dagger}\right\}=$ $0 ; \quad\left\{c_{x}^{\dagger}, c_{y}\right\}=\delta_{x y}$. The corresponding bosonic system is obtained by using the Jordan-Wigner transformation. An ordering of the lattice points is defined: $l=x+y \cdot L($ in $d=2)$, and the fermionic operators are expressed as follows:

$$
c_{x}^{\dagger}=\sigma_{1}^{3} \cdot \sigma_{2}^{3} \cdots \sigma_{l-1}^{3} \sigma_{l}^{+} ; \quad c_{x}=\sigma_{1}^{3} \cdot \sigma_{2}^{3} \cdots \sigma_{l-1}^{3} \sigma_{l}^{-} ; n_{x}=c_{x}^{\dagger} c_{x}=\frac{1}{2}\left(\sigma_{l}^{3}+1\right) .
$$

This preserves the anti-commutation relations. We use the (fermion) occupation number basis $\left(n_{x}|0\rangle=0, n_{x}|1\rangle=|1\rangle\right.$, with 0 denoting an unoccupied and 
1 an occupied site) to construct $\mathcal{Z}_{\mathrm{f}}$ by splitting the Hamiltonian into different parts and invoking the Suzuki-Trotter formula. With some algebra to keep track of the negative signs, the transfer matrix between the adjacent timeslices can be expressed through the following $4 \times 4$ plaquette interactions (the state labels at sites $x$ and $y$ denote $|00\rangle,|01\rangle,|10\rangle,|11\rangle)$ :

$$
\begin{aligned}
\mathrm{e}^{-S\left[n_{1}, n_{2}, n_{3}, n_{4}\right]} & =\left\langle n_{1} n_{2}\left|\mathrm{e}^{\epsilon \frac{\mathrm{t}}{2}\left(c_{x}^{\dagger} c_{y}+c_{y}^{\dagger} c_{x}\right)-\epsilon V\left(n_{x}-\frac{1}{2}\right)\left(n_{y}-\frac{1}{2}\right)}\right| n_{3} n_{4}\right\rangle \\
& =\mathrm{e}^{\frac{\epsilon V}{4}}\left[\begin{array}{cccc}
\mathrm{e}^{-\epsilon V / 2} & 0 & 0 & 0 \\
0 & \cosh (\epsilon \mathrm{t} / 2) & \Sigma \sinh (\epsilon \mathrm{t} / 2) & 0 \\
0 & \Sigma \sinh (\epsilon \mathrm{t} / 2) & \cosh (\epsilon \mathrm{t} / 2) & 0 \\
0 & 0 & 0 & \mathrm{e}^{-\epsilon V / 2}
\end{array}\right]
\end{aligned}
$$

The structure of the transfer matrix is very similar to the one of the quantum spins, the main difference is the string operator $\Sigma=\sigma_{l+1}^{3} \cdot \sigma_{l+2}^{3} \cdots \sigma_{m-1}^{3}$ between the sites $x=l$ and $x+\hat{i}=m$, where $l<m$ in the lattice ordering. This allows the rewriting of $\mathcal{Z}_{\mathrm{f}}$ in the occupation number basis $\left[\left\{n_{x}=0,1\right\}\right]$ as

$$
\mathcal{Z}_{\mathrm{f}}=\sum_{\{n\}} \operatorname{sign}[\{n\}] \mathrm{e}^{-S[\{n\}]} .
$$

Some example configurations which contribute to $\mathcal{Z}_{\mathrm{f}}$ are shown in Fig. 2 Without the sign factor, the weights of the resulting bosonic system are identical to that of the XXZ-Hamiltonian:

$$
H=\sum_{x, \hat{i}=1,2}\left[\mathrm{t}\left(S_{x}^{1} \cdot S_{x+\hat{i}}^{1}+S_{x}^{2} \cdot S_{x+\hat{i}}^{2}\right)+\mathrm{V} S_{x}^{3} \cdot S_{x+\hat{i}}^{3}\right],
$$

which reduces to the anti-ferromagnet for $\mathrm{t}=\mathrm{V}=J$. The $\mathcal{Z}_{\mathrm{f}}$ is decomposed in terms of bonds, in addition to spins:

$$
\mathcal{Z}_{\mathrm{f}}=\sum_{[n, b]} \operatorname{sign}[n, b] \exp ([-S[n, b]])
$$

Interestingly, in the limit $\mathrm{t}=\mathrm{V}$ the only breakups that are needed are the $A$ and the $B$ breakup, with same probabilities as derived in Fig. 1 (right). Fig 2 shows a typical fermion configuration, and a particular set of breakups, and how clusters are identified. Cluster flips are operations $\left\{n_{k} \leftrightarrow 1-n_{k} \mid n_{k} \in \mathcal{C}_{i}\right\}$, where $\mathcal{C}_{i}$ is the $\mathrm{i}$-th cluster. Cluster flips give rise to new worldlines significantly different from their parent ones, but carry the same weights.

The crucial consequence of these cluster rules is that the sign of the whole configuration factorizes into a product of the signs associated with each individual cluster. An example is already seen in Fig 2 (right). The clusters which can change the sign of the configuration are called merons. It is possible to reach a reference configuration by flipping clusters appropriately. In this example, the configuration in Fig 2 (left) is the reference configuration, which has a positive sign $\left(\operatorname{sign}_{\mathcal{C}_{i}}=1\right)$ for all clusters $i$. When a cluster is flipped, its contribution to sign is $\operatorname{sign}_{\mathcal{C}_{i}}=1(-1)$ if the quantity $N_{w}+N_{h} / 2$ is odd (even). $N_{w}$ is the temporal winding number of the cluster, while $N_{h}$ is the number 

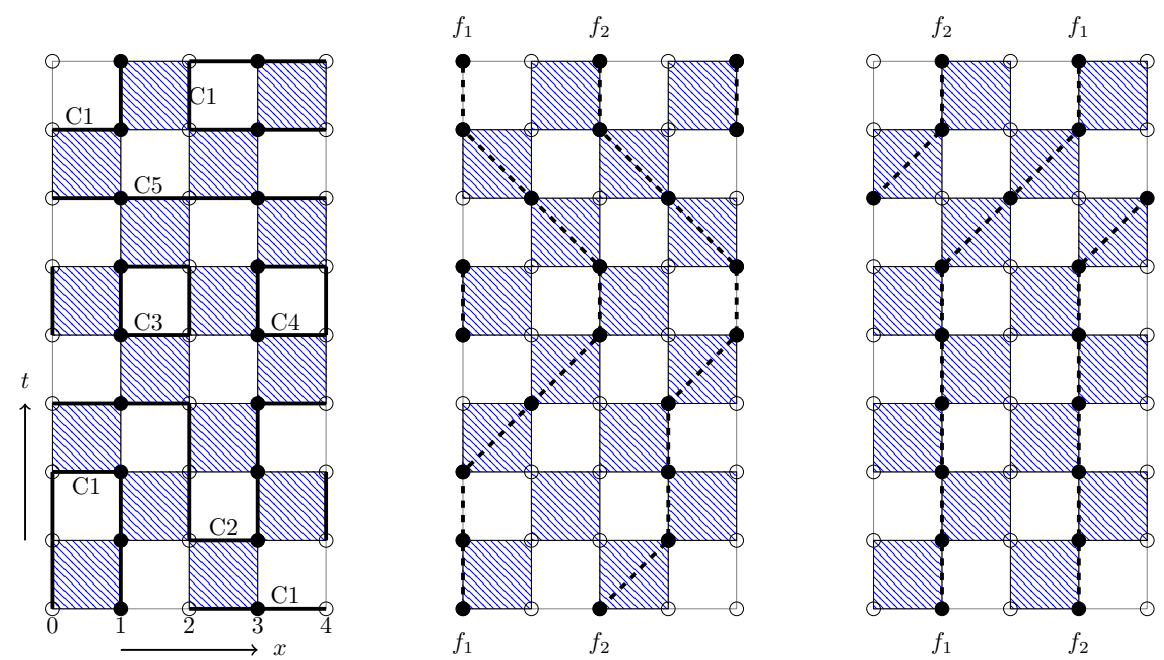

Fig. 2 Example configurations which contributes to $\mathcal{Z}_{\mathrm{f}}$. (left) The two fermions $f_{1}$ and $f_{2}$ are static during their entire Euclidean time-evolution, and trace out vertical worldlines. For $t=J$, only $A$ (vertical) and $B$ (horizontal) bonds are required. The figure shows one bond configuration. The oriented clusters are constructed by starting from a single point and following the bonds - upwards (downwards) for a filled (empty) site for $A$-breakups, sideways for a filled (empty) site touching $B$-breakups. This choice of breakups gives rise to 5 different clusters, as marked in the figure. (middle) On flipping clusters marked 1, 3 and 4 we obtain a completely different world-line configuration, but one where the fermions do not exchange positions. (right) Flipping cluster 5 gives rise to a configuration where the fermions interchange positions, and hence due to the anticommutation relations, this configuration has an overall negative sign compared to the one on the left. The cluster 5 is a meron.

of spatial hops 32. The meron concept allows exact pairing of even and odd signs:

$$
\begin{aligned}
\mathcal{Z}_{\mathrm{f}} & =\sum_{[n, b]} \overline{\operatorname{sign}[b]} \exp (-S[n, b])=\sum_{[b], \text { zero-meron }} 2^{N_{\mathcal{C}}} \exp (-S[b]) ; \\
\overline{\operatorname{sign}[b]} & =\frac{1}{2^{N_{\mathcal{C}}}} \sum_{\text {clusterflips }} \operatorname{sign}[n, b] .
\end{aligned}
$$

$\overline{\operatorname{sign}[b]}=0$ if at least one of the clusters is a meron. Further, the existence of the reference configuration with a positive Boltzmann weight, guarantees that the unpaired configurations all carry positive weight, and can be reached by flipping clusters from an initial configuration which has zero-merons. The Monte Carlo sampling proceeds by generating configurations which only lie in the zero-meron sector. If a proposed flip gives rise to meron cluster, it is rejected. Thus, one is able to completely solve the sign problem with the meron concept.

Let us emphasize that for generic interactions the meron concept does not hold, and whether a cluster is a meron depends on the orientation of other 

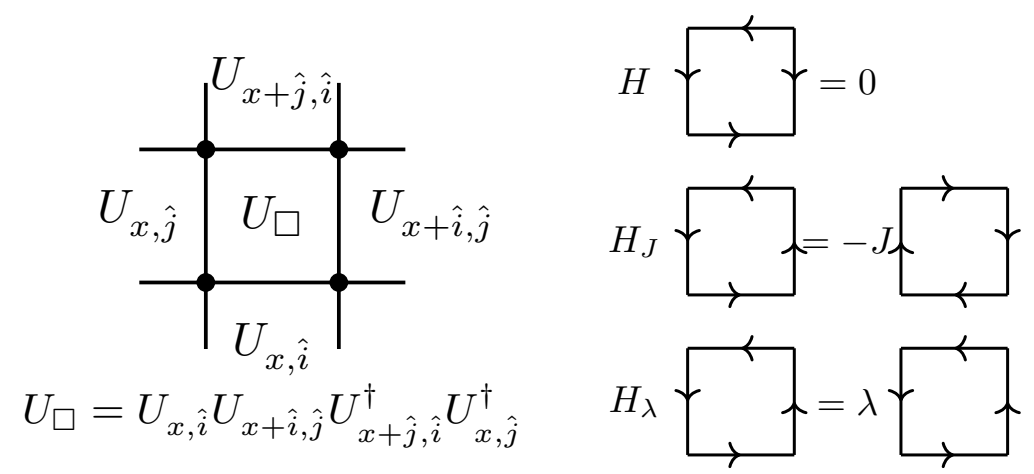

Fig. 3 (left) Layout of the plaquette, and the four spin interaction. (right) The action of the operators in the Hamiltonian on the possible plaquette states. The $U_{\square}\left(U_{\square}^{\dagger}\right)$ term flips a clockwise (anti-clockwise) oriented plaquette to an anti-clockwise (clockwise) oriented one, and annhiliates the remaining 14 possible states. This is the $J$ - term of the Hamiltonian, and is an off-diagonal operator. The $\lambda$-term counts the total number of flippable (both in the clockwise and anti-clockwise orientation) plaquettes.

clusters. Therefore, much more (exponential) computational effort is needed to identify the merons. However, there is a large class of interactions which can be solved with the meron concept, and we refer the reader to 31 for a review.

\section{Pure Gauge Theories}

Cluster algorithms for gauge theories run into problems since the relevant variables that need to be updated get frustrated, and very often the clusters occupy the entire volume. The resulting algorithm is not much better than a local update algorithm. One direction of research tried to identify the relevant degrees of freedom, which may or may not be identical to the degrees of freedom in which the action is constructed. For example, for a $\phi^{4}$ theory, the $\phi$ field can be separated into an Ising $\left(Z_{2}\right)$ variable and a modulus variable. The $Z_{2}$ degrees of freedom were updated using a cluster algorithm while the modulus of the $\phi$ field was updated with a local update algorithm [37], which resulted in an efficient algorithm with a low $(z<1)$ dynamical exponent. Similar methods have been applied to the $S U(2)$ lattice gauge theory [38, 39] targeting the Polyakov loop as the embedded degree of freedom. While some success on the theories with one and two time-slices was reported, these methods do not work well for larger lattices.

A completely different formulation of gauge theories, the quantum link model formulation [40,41,42, which realizes continuous gauge symmetries with finite dimensional Hilbert spaces and generalizes the Wilson construction of lattice gauge theories, has recently been more amenable to simulation 
with cluster algorithms. As an illustrative example, we will describe the $U(1)$ quantum link model (QLM). The degrees of freedom of this Abelian lattice gauge theory are quantum links, $U_{x, \hat{i}}$ and electric fluxes, $E_{x, \hat{i}}$, both of which are operators defined on the bonds connecting neighboring sites, $x$ and $x+\hat{i}$ and $\hat{i}=1,2$ in $d=2$. These operators satisfy canonical commutation relations:

$$
\left[E_{x, \hat{i}}, U_{y, \hat{j}}\right]=U_{x, \hat{i}} \delta_{x y} \delta_{i j} ;\left[E_{x, \hat{i}}, U_{y, \hat{j}}^{\dagger}\right]=-U_{x, \hat{i}}^{\dagger} \delta_{x y} \delta_{i j} ;\left[U_{x, \hat{i}}, U_{y, \hat{j}}^{\dagger}\right]=2 E_{x, \hat{i}} \delta_{x y} \delta_{i j},
$$

The operators $E, U$ can be chosen to be the components of a spin- $S$ object: $U=S^{+}, U^{\dagger}=S^{-}, E=S^{3}$. The parameter $S$ can be thought of regulating the local Hilbert space dimension. In the limit $S \rightarrow \infty$ [46, these models reproduce the Hamiltonian formulation of Wilson gauge theories [47. The Hamiltonian we will be interested in only contains operators which commute with the Gauss' Law:

$$
H=-J \sum_{\square}\left(U_{\square}+U_{\square}^{\dagger}\right)+\lambda \sum_{\square}\left(U_{\square}+U_{\square}^{\dagger}\right)^{2} ; G_{x}=\sum_{i}\left(E_{x, x+i}-E_{x-i, x}\right)
$$

In addition, we could have included any function of the electric fluxes, and in particular, the kinetic energy of the gauge fields $\sum_{x, i} E_{x, i}^{2}$. When the quantum link operators are considered in the spin- $\frac{1}{2}$ representation, this model has several interesting physical applications. In $(2+1)-\mathrm{d}$, the physics of this model is relevant for the understanding the behavior of low-temperature frustrated magnets [43, and spin-liquid phases [44,45]. A close cousin of this model is the quantum dimer model (QDM), especially well-known in condensed matter physics as a toy model to describe certain aspects of superconductivity [48,49, 50]. Therefore, we continue with the gauge links in the spin- $\frac{1}{2}$ representation, and hence ignore the kinetic energy term of the gauge links, which is a constant. The local link Hilbert space is 2-dimensional, and in the electric flux basis they are denoted by upward (downward) arrows for $E=\frac{1}{2}\left(-\frac{1}{2}\right)$ for vertical links, and right (left) arrow for $E=\frac{1}{2}\left(-\frac{1}{2}\right)$ for horizontal links. The action of the Hamiltonian on the electric flux states is shown in Fig 3.

Specifying the Gauss' Law further specifies which basis states can contribute to the partition function, and which not. For the QLM, the Gauss' Law is chosen as $G_{x}|\psi\rangle=0$, for every site $x$, for every eigenstate $|\psi\rangle$ of the Hamiltonian. The QDM chooses a different set of states, which are specified by $G_{x}|\psi\rangle=(-1)^{x_{1}+x_{2}}|\psi\rangle$. Physically, this implies that the vacuum selected by the QLM is charge neutral at each vertex, while the QDM chooses a vacuum with staggered positive and negative unit charges. The link states allowed at the vertex for the QLM is shown in Fig 4 (left) and for the QDM in Fig 4 (right). The Gauss' Law generates the gauge transformations, which can be generically denoted as $V=\prod_{x} \exp \left(i \theta_{x} G_{x}\right)$, where $\theta_{x} \in(0,2 \pi]$ is the parameter of the transformation. The Hamiltonian is invariant under this transformation: $\widetilde{H}=V^{\dagger} \cdot H \cdot V=H$, since $\left[G_{x}, H\right]=0$, for all x. Physically, $G_{x}$ labels different super-selection sectors of the theory which do not mix under unitary dynamics. 

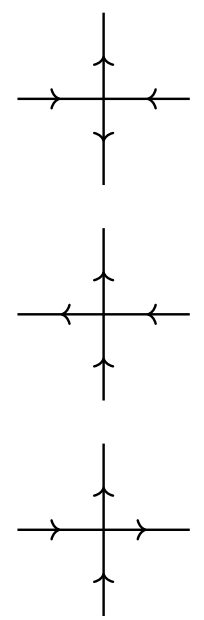
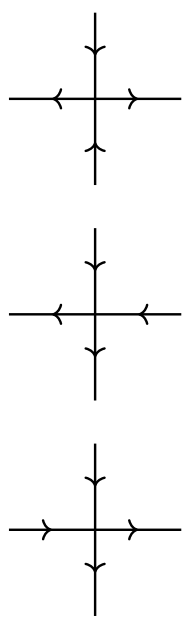
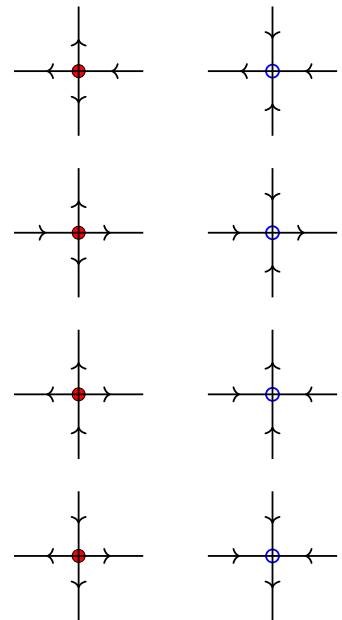

Fig. 4 (left) The Gauss' Law condition for the QLM, in $d=2$, this allows 6 possible states on each vertex, while (right) the Gauss' Law condition for the QDM in $d=2$ allows for 4 states for each vertex. The red circle represents $Q=+1$, while the blue circle the $Q=-1$, which are distributed in a staggered fashion on the square lattice.

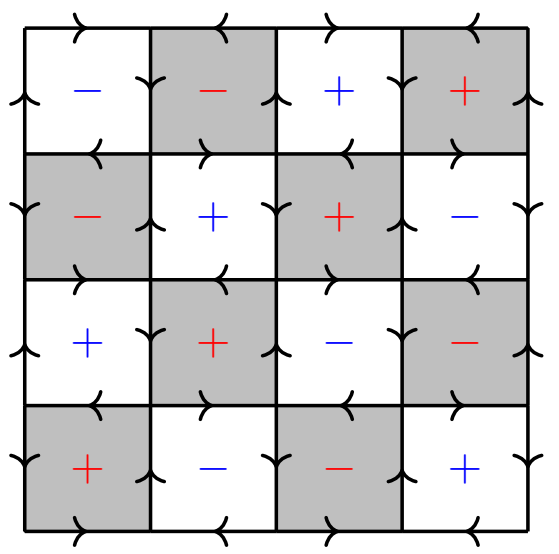

Fig. 5 Mapping of an electric flux configuration (shown with arrows on the links) to a height configuration (shown with + and - variables at the centers of the plaquettes). The + and - are placeholders for $0,1\left( \pm \frac{1}{2}\right)$ on A (B) sub-lattices. Every time a flux pointing right or upwards (corresponding to $E=\frac{1}{2}$ ) is crossed, the height variable is changed, while it remains unchanged if a left or downward pointing flux (corresponding to $E=-\frac{1}{2}$ ) is crossed. The configuration shown has all plaquettes flippable, and the corresponding height variables are in their reference configuration. 
The partition function of this model is defined by projecting the eigenstates of the Hamiltonian into a specified Gauss' Law sector, $\mathcal{Z}=\operatorname{Tr}\left[\exp (-\beta H) \mathcal{P}_{G}\right]$. The projection operator $\mathcal{P}_{G}=\prod_{x} G_{x}$, therefore constrains the Hilbert space further. To construct the cluster algorithm, we note that we can divide the Hamiltonian into two parts $H=H_{A}+H_{B}$, such that the all terms in each part mutually commute. Thus, the Trotterization divides the lattice into even and odd sub-lattices, such that at a given time-step only a single sub-lattice needs to be updated. For a fixed $\beta=\epsilon N$, we have a two-step transfer matrix

$$
\mathcal{Z}=\operatorname{Tr}\left[\left(T_{A} T_{B}\right)^{N} P_{G}\right] .
$$

The single plaquette transfer matrix can be expressed as

$$
T_{\square}=1+\left(U_{\square}+U_{\square}^{\dagger}\right) \mathrm{e}^{-\epsilon J \lambda} \sinh (\epsilon J)+\left(U_{\square}+U_{\square}^{\dagger}\right)^{2}\left[\mathrm{e}^{-\epsilon J \lambda} \cosh (\epsilon J)-1\right]
$$

The resulting transfer matrix is 16-dimensional (i.e. a $16 \times 16$ matrix), and it is easy to read off the Boltzmann weights from this equation. For plaquettes which are not flippable, only the diagonal element is unity, and all other offdiagonal elements are zero. For the two flippable plaquette configurations, the diagonal contribution has the weight $\mathrm{e}^{-\epsilon \lambda} \cosh (\epsilon J)$ while the off-diagonal elements (which indicate the plaquette flips) carry the weight $\mathrm{e}^{-\epsilon \lambda} \sinh (\epsilon J)$.

The idea behind the construction of the cluster algorithm is to dualize the gauge theory in $(2+1)-d$, which gives rise to a $\mathbb{Z}(2)$ quantum height model in $(2+1)-\mathrm{d}$. The dualization is an exact rewriting of the partition function in terms of new degrees of freedom which are $\mathbb{Z}(2)$ degrees of freedom located at dual sites. As shown in Fig 5, every flux configuration can be mapped to a height configuration. A configuration of quantum height variables is assigned the values $h_{\tilde{x}}^{A}=0,1$ and $h_{\tilde{x}}^{B}= \pm \frac{1}{2}$. located at the dual sites $\tilde{x}=\left(x_{1}+\frac{1}{2}, x_{2}+\frac{1}{2}\right)$, and is associated with a flux configuration

$$
E_{x, \hat{i}}=\left[h_{\tilde{x}}^{X}-h_{\tilde{x}+\hat{i}-\hat{1}-\hat{2}}^{X^{\prime}}\right] \bmod 2= \pm \frac{1}{2} ; \quad X, X^{\prime} \in\{A, B\} .
$$

The modulo function acts by adding or subtracting 2 until the result is in the desired range $(-1,1]$. We note that while for each height configuration there is exactly one flux configuration, the reverse is not true. It can be shown that there are exactly two height configurations for each flux configuration. In addition we note that the mapping to the height variables only works when (a) there are no charges at the lattice sites and (b) when charges $Q= \pm 2$ are located on the lattice sites. This explicitly indicates the $\mathbb{Z}(2)$ nature of the height variables.

Before deriving the cluster rules, it is useful to understand the Boltzmann weights of the configurations in terms of the height variables, as shown Fig 6. In terms of the height variables, the transfer matrix gives rise to a 6height interaction between the heights $m_{1}, \cdots, m_{6}$. Of these, $m_{1}, m_{2}, m_{3}, m_{4}$ live on a timeslice t, $m_{5}$ lives on the timeslice $\mathrm{t}-1$ and $m_{6}$ on $\mathrm{t}+1$. Depending on the values of $m_{1}, m_{2}, m_{3}, m_{4}$, the height $m_{5}$ can undergo a transition to $m_{6}$ (corresponding to a plaquette flip), or not. In this language if 


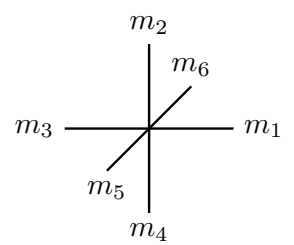

$m_{1}, m_{2}, m_{3}, m_{4}$ height variables

lives at timeslice $\mathrm{t}$

$m_{5}\left(m_{6}\right)$ lives at timeslice $\mathrm{t}-1(\mathrm{t}+1)$

Bonds for top-right panel

$A$ join $m_{5}$ and $m_{6}$

$B$ keep $m_{5}$ and $m_{6}$ separate

Bonds for bottom-right panel

$A$ join $m_{1}, m_{2}, m_{3}, m_{4}$

$B$ keep $m_{1}, m_{2}, m_{3}, m_{4}$ separate
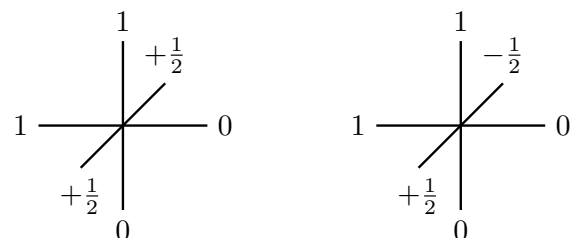

$\mathrm{e}^{-\epsilon J \lambda} \sinh (\epsilon J)$ $B$

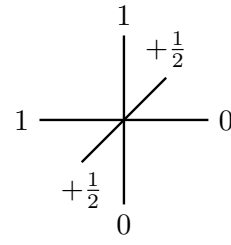

$\mathrm{e}^{-\epsilon J \lambda} \cosh (\epsilon J)$

$A+B$

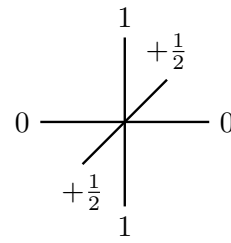

1

Fig. 6 (left top) Layout of the height variables across the timeslices $t-1, t$ and $t+1$. (left bottom) Two kinds of bonds are enough to solve the linear equations: the A-breakup joins heights, while the B-breakup keeps the heights separate. Different scenarios need to be considered - out-of-plane (temporal) bonds, and in-plane (spatial) bonds. (right top) Cluster rules for temporal bonds: with $m_{1}, m_{2}, m_{3}, m_{4}$ in a reference configuration and $m_{5}=m_{6}$, both A- and B-breakups can contribute, while if $m_{5} \neq m_{6}$ then only the B-breakup contributes. To satisfy detailed balance, we must have $P_{A}=A /(A+B)=\mathrm{e}^{-\epsilon J \lambda} / \cosh (\epsilon J)$ and $P_{B}=\tanh (\epsilon J)$. In the case when $m_{5}=m_{6}$ and $m_{1}, m_{2}, m_{3}, m_{4}$ are not in a reference configuration, then we have to bind $m_{5}$ and $m_{6}$ with probability 1 , otherwise a forbidden configuration will be generated. (right bottom) Spatial breakups: if $m_{5} \neq m_{6}$ then connect $m_{1}, m_{2}, m_{3}, m_{4}$ with probability 1 , to prevent the generation of a forbidden configuration. If $m_{5}=m_{6}$ then two different cases can occur: if $m_{1}, m_{2}, m_{3}, m_{4}$ are in a reference configuration, then the Boltzmann weight is $\mathrm{e}^{-\epsilon J \lambda} \cosh (\epsilon J)$ and we can apply either the $\mathrm{A}$, or the B-breakup. Otherwise, the weight is 1 and only B breakup is applied $\left(m_{5}\right.$ and $m_{6}$ are not bound together). Solving the equations, we get $P_{A}=1-\mathrm{e}^{-\epsilon J \lambda} / \cosh (\epsilon J)$ and $P_{B}=1-P_{A}$.

$\left(m_{1}, m_{2}, m_{3}, m_{4}\right)=(1,1,0,0)$ or $(0,0,1,1)$ the plaquette is flippable, and form the reference configurations. The weights of these configurations and the derivation of the cluster rules is illustrated in Fig 6 and the figure caption.

With these cluster rules implemented in terms of the height variables, the super-selection sectors which have charges $Q_{x}= \pm 1$ are never generated. However, it is still possible generate charges $Q_{x}= \pm 2$, since they are compatible with the height representation. Therefore, in addition to the cluster rules for the Hamiltonian, the ones for the Gauss' Law need to additionally implemented. To implement the Gauss' Law note that we need to consider four height variables meeting around a site: the top-left and the bottom-right belong to a one sub-lattice (say A), and top-right and bottom left belong to the other sub-lattice (B). It can be easily verified that if the site contains a 
charge \pm 2 , then both the height variables in both sub-lattices are locally out of their reference configuration. Thus, this situation should always be avoided. This can be implemented as follows: when updating the A sub-lattice at a given site, it is checked if the corresponding sites of the B sub-lattice are out of the reference configuration. If this is the case then we bind the two height variables together, so that they are always flipped together, and a forbidden configuration is not generated. If the relevant height variables in the B sublattice are in a reference configuration then no additional bonds are put on the A sub-lattice heights. An identical procedure is followed while updating the other sub-lattice. It is sufficient to check this on a single timeslice, since the Gauss' Law commutes with the Hamiltonian.

We emphasize that this cluster algorithm for the gauge theory is somewhat different from the usual type of cluster algorithms since clusters are separately built on each sub-lattice depending on the values of frozen height variables in the other sub-lattice. In the next step, the frozen sub-lattice is updated. This algorithm was first implemented in [51] to study the physics of the $\mathrm{U}(1)$ QLM and it uncovered new phases of confined gauge theories which break translation and charge conjugation symmetry [52].

It is interesting to note that, even though this algorithm is very efficient on the QLM, extending this to the QDM is not as useful. Since the Hamiltonian is the same, the same cluster rules can be applied. The problem is however with the different Gauss' Law, which now frustrates the reference configuration. Due to the absence of the reference configuration in the chosen basis of the quantum height model, the clusters grow quite large to occupy about $80-90 \%$ of the space-time volume, which makes the cluster algorithm not much better than a local update Metropolis algorithm. However, a combination of both these algorithms was used to study the physics of the QDM [53,54] and reliably extract the phase diagram at zero temperature. For the QDM certain other computational methods have been reported [55,56], including certain novel effective theory approaches $[57$.

\section{Conclusions and Outlook}

Cluster algorithms are extremely efficient tools which are very useful to speed up the Monte Carlo sampling of strongly interacting systems wherever they are applicable. These algorithms act by placing bonds between degrees of freedom which are correlated, and build up patches in configuration space which can be flipped. The flipping process gives rise to a new configuration which is very different from the parent one, but has the same Boltzmann weight. Thus, the correlation in between the subsequent Monte Carlo configurations are removed, since the clusters themselves are physical degrees of freedom. In the case of fermions, configurations often come with negative signs associated with fermions interchanging their respective positions. A novel cluster algorithm - the meron algorithm, is able to analytically cancel clusters which are responsible for the negative worldlines, and only operate in the Hilbert space 
which contribute non-trivially to the partition function. Cluster algorithms for gauge theories are even more challenging than other systems since one has to identify proper degrees of freedom which can be used to build clusters that do not occupy the whole space-time volume. In the case for certain quantum link models, this has been achieved by dualizing the original Hamiltonian, and rewriting it exactly in terms of dual quantum height variables. The resulting cluster algorithm is very efficient. Further, for gauge theories, the Gauss' Law needs to be implemented, which may or may not be possible with the cluster rules that allow the sampling the full Hilbert space. For example, in the case of QLM where the vacuum is charge neutral, this is not a problem, but the QDM which has a staggered background charge this does not work so well, and the clusters become large. This can be understood as the lack of a reference configuration for the QDM, which is an essential ingredient for the success of a cluster algorithm.

Demanding the existence of reference configurations, it is possible to construct models which are guaranteed to have efficient cluster algorithm. Such Hamiltonians are often called designer Hamiltonians [58, and can be studied in any space-time dimensions. Since the exact form of the microscopic interactions is unimportant to study physics associated with breaking of symmetries across thermal or quantum phase transitions, thanks to universality, cluster algorithms can be used to study a wide range of physical phenomena in naturally occurring strongly correlated systems.

In the past few years, there has been renewed interest with cluster and meron algorithms. In the context of classical spin models, the meron algorithm is being used to study the topological charge and the vorticity properties of non-linear sigma models [59]. For lattice fermions, the meron algorithm has been used to study a Hamiltonian spin-half lattice fermions that displays symmetry enhancement for certain coupling regimes 60. In the context of gauge theories, the dualization technique has been extended to a non-Abelian $S U(2)$ quantum link model on the honeycomb lattice and an efficient cluster algorithm has been constructed in terms of the dual height variables 61. This cluster algorithm operates on a four sub-lattice structure, where the clusters are built using the heights on one sub-lattice, while the heights on the other three sub-lattices decide the nature of bonds. This study identified more crystalline confined phases in non-Abelian theories, as well as showed fractionalization of a $Z(2)$ flux.

Another very novel and interesting development with cluster algorithms is the simulation of strongly correlated systems in real-time and far out of equilibrium. The first such study considered interesting initial states which are in contact with a thermal reservoir, and are completely driven via dissipation. The authors of [62] devised a cluster algorithm to simulate the Lindblad evolution in an anti-ferromagnetic initial state. More extensive studies revealed what kinds of measurements give rise to sign problems, and which measurements did not [63]. Transport phenomena in strongly correlated spin- $\frac{1}{2}$ driven via Lindblad dynamics was studied using cluster algorithms in [64]. 
Acknowledgements This article is written in the memory of the late Pushan Majumdar. Pushan taught me the intricacies of the multi-level algorithm 65, 66, a highly efficient Monte Carlo method which greatly accelerates the simulation of pure Wilson-type gauge theories. Besides, I would like to acknowledge Pushan for the many discussions on physics, his advice on parallel and GPU programming that we have had, and for the many cups of coffee he would prepare after lunch.

I would like to thank Shailesh Chandrasekharan and Uwe-Jens Wiese for teaching me so much about cluster and meron algorithms. I would also like to acknowledge Saumen Datta, Fu-Jiun Jiang, Kieran Holland, Emilie Huffman, Ferenc Niedermayer, Stefan Schaefer, Arnab Sen, Rainer Sommer, Urs Wenger, and Ulli Wolff for various discussion on improved algorithms.

\section{References}

1. J. Cardy, Conformal Field Theory and Statistical Mechanics, Les Houches Summer School on Exact Methods in Low-Dimensional Statistical Physics and Quantum Computing (2008).

2. A. Pelissetto, E. Vicari, Critical Phenomena and renormalization group theory, Phys. Rept., 368, 549 (2002).

3. M. Moshe. J. Zinn-Justin, Quantum Field Theory in the Large-N limit: A Review, Phys. Rept., 385, 69 (2003).

4. J. Maldacena, The Large-N Limit of Superconformal Field Theories and Supergravity, Intl. J. of Theo. Phys., 38, 1113 (1999).

5. R. Rattazzi, V. Rychkov, E. Tonni, A. Vichi, Bounding scalar operator dimensions in $4 D$ CFT, JHEP 12, 31 (2008).

6. S. Hellerman, D. Orlando, S. Reffert, M. Watanabe, On the CFT Operator Spectrum at Large Global Charge, JHEP 17, 12 (2015).

7. U. Schollwoeck, The density-matrix renormalization group in the age of matrix product states, Ann. Phys. 326, 96 (2011).

8. N. Metropolis, A. Rosenbluth, M. Rosenbluth, A. Teller, E. Teller, Equation of State Calculations by Fast Computing Machines, J. Chem. Phys. 21, 1087 (1953).

9. D. Landau, K. Binder, A Guide to Monte Carlo Simulations in Statistical Physics, Cambridge University Press (2014).

10. M. Newman, G. Barkema, Monte Carlo Methods in Statistical Physics, Clarendon Press (1999).

11. P. W. Kasteleyn, C. M. Fortuin, Phase transitions in lattice systems with random local properties, J. Phys. Soc. Jpn. Suppl., 26, 11 (1969).

12. C. M. Fortuin, P. W. Kasteleyn, On the random-cluster model. I. Introduction and relation to other models, Physica 57, 536 (1972).

13. R. H. Swendsen, J. -S. Wang, Non-universal critical dynamics in Monte Carlo simulations, Phys. Rev. Lett. 58(2), 86 (1987).

14. F. Niedermayer, General Cluster Updating Method for Monte Carlo Simulations, Phys. Rev. Lett. 61, 2026 (1988).

15. U. Wolff, Collective Monte Carlo updating for spin systems, Phys. Rev. Lett.62(4), 361 (1989).

16. F. Niedermayer, Cluster Algorithms, Advances in Computer Simulations, Lecture Notes in Physics, Vol 501, 36 (1997).

17. W. Krauth, Cluster Monte Carlo Algorithms, New Optimization Algorithms in Physics, (2003).

18. H. G. Evertz, G. Lana, M. Marcu, Cluster algorithm for vertex models, Phys. Rev. Lett. 70,875 (1993).

19. H. G. Evertz, M. Marcu, The Loop-Cluster Algorithm for the Case of the 6 Vertex Model, Nucl. Phys. Proc. Suppl. 30, 277 (1993).

20. B. B. Beard, U.-J. Wiese, Simulations of Discrete Quantum Systems in Continuous Euclidean Time, Phys. Rev. Lett. 77, 5130 (1996).

21. A. W. Sandvik, J. Kurkijärvi, Quantum Monte Carlo simulation method for spin systems, Phys. Rev. B 43, 5950 (1991). 
22. O. F. Syljuåsen, A. W. Sandvik, Quantum Monte Carlo with Directed Loops, Phys. Rev. E 66, 046701 (2002).

23. H. G. Evertz, The loop algorithm, Advances in Physics 52 (2003).

24. N. V. Prokof'ev, B. V. Svistunov, Worm Algorithms for Classical Statistical Models, Phys. Rev. Lett. 87, 160601 (2001).

25. W. Heisenberg, On the theory of ferromagnetism, Zeit. für Phys., 49 (9), 619 (1928).

26. M. Suzuki, Relationship between d-dimensional quantum spin system and $(d+1)$ dimensional Ising systems, Prog. Theor. Phys., 56, 1454 (1976).

27. M. Troyer, U.-J. Wiese, Computational Complexity and Fundamental Limitations to Fermionic Quantum Monte Carlo Simulations, Phys. Rev. Lett. 94, 170201 (2005).

28. R. Blankenbecler, D. J. Scalapino, R. L. Sugar, Monte Carlo calculations of coupled boson-fermion systems. I, Phys. Rev. D. 24 (8), 2278 (1981).

29. F. Assaad, H. G. Evertz, World-line and determinantal quantum Monte Carlo Methods for spins, phonons and electrons, Lecture Notes in Physics, Vol. 739, pp. 277-356, Springer, Berlin Heidelberg (2008).

30. S. Duane, A. D. Kennedy, B. J. Pendleton, D. Roweth, Hybrid Monte Carlo, Phys. Lett. B 195 (2), 216 (1987).

31. S. Chandrasekharan, J. Cox, J. C. Osborn, U.-J. Wiese, Meron cluster approach to systems of strongly correlated electrons, Nucl. Phys. B 673, 405 (2003).

32. S. Chandrasekharan, U.-J. Wiese, Meron cluster solution of fermion sign problems, Phys. Rev. Lett. 83, 3116 (1999).

33. S. Chandrasekharan, The Fermion bag approach to lattice field theories, Phys. Rev. D 82, 025007 (2010)

34. E. Huffman, S. Chandrasekharan, Fermion bag approach to Hamiltonian lattice field theories in continuous time, Phys. Rev. D 96, 114502 (2017).

35. M. Boninsegni, N. V. Prokof'ev, B. V. Svistunov, Worm algorithm and diagrammatic Monte Carlo: a new approach to continuous-space path integral Monte Carlo simulations, Phys. Rev. E 74, 036701 (2006).

36. K. van Houcke, E. Kozik, N. V. Prokof'ev, B. V. Svistunov, Diagrammatic Monte Carlo, Phys. Procedia 6, 95 (2010)

37. R. Brower, P. Tamayo, Embedded dynamics for $\phi^{4}$ theory, Phys. Rev. Lett. 62, 1087 (1989).

38. R. Ben-Av, H. G. Evertz, M. Marcu, S. Solomon, Critical acceleration of finite temperature SU(2) gauge simulations, Phys. Rev. D 44, 2963 (1991).

39. W. Kerler, T. Metz, Ising embedding for cluster algorithms in finite temperature SU(2) gauge theory, Phys. Rev. D 50, 7082 (1994).

40. D. Horn, Finite Matrix Models with Continuous Local Gauge Invariance, Phys. Lett. B 100, 149 (1981)

41. P. Orland, D. Rohrlich, Lattice gauge magnets: Local isospin from spin, Nucl. Phys. B 338,647 (1990).

42. S. Chandrasekharan, U.-J. Wiese, Quantum Link Models: A Discrete Approach to Gauge Theories, Nucl. Phys. B 492, 455 (1997).

43. N. Shannon, G. Misguich, K. Penc, Cyclic exchange, isolated states and spinon deconfinement in an XXZ Heisenberg model on the checkerboard lattice, Phys. Rev. B 69, 220403 (2004).

44. M. Hermele, M. Fisher, L. Balents, Pyrochlore Photons: The U (1) Spin-Liquid in a S=1/2 Three Dimensional Frustrated Magnet, Phys. Rev. B 69064404 (2004).

45. L. Balents, Spin liquids in frustrated magnets, Nature 464, 199 (2010).

46. B. Schlittgen, U.-J. Wiese, Low-energy effective theories of quantum spin and quantum link models, Phys. Rev. D 63, 085007 (2001).

47. J. B. Kogut, L. Susskind, Hamiltonian Formulation of Wilson's Lattice Gauge Theories, Phys. Rev. D 11, 395 (1975).

48. D. S. Rokhsar, S. A. Kivelson, Superconductivity and the Quantum Hard-Core Dimer Gas, Phys. Rev. Lett. 61, 2376 (1988).

49. S. Sachdev, Spin-Peierls ground states of the quantum dimer model: A finite-size study, Phys. Rev. B 40, 5204 (1989).

50. R. Moessner, K. S. Raman, Quantum Dimer Models, Lecture Notes, Trieste 2007 (arXiv:0809.3051). 
51. D. Banerjee, F.-J. Jiang, P. Widmer, U.-J. Wiese, The (2 + 1)-d U(1) quantum link model masquerading as deconfined criticality, J. Stat. Mech. 1312, P12010 (2013).

52. D. Banerjee, P. Widmer, F.-J. Jiang, U.-J. Wiese, Crystalline Confinement, PoS LATTICE2013, 333 (2014).

53. D. Banerjee, M. Bögli, C. P. Hofmann, F.-J. Jiang, P. Widmer, U.-J. Wiese, Interfaces, Strings, and a Soft Mode in the Square Lattice Quantum Dimer Model, Phys. Rev. B 90, 24, 245143 (2014)

54. D. Banerjee, M. Bögli, C. P. Hofmann, F.-J. Jiang, P. Widmer, U.-J. Wiese, FiniteVolume Energy Spectrum, Fractionalized Strings, and Low-Energy Effective Field Theory for the Quantum Dimer Model on the Square Lattice, Phys. Rev. B 94, 11, 115120 (2016).

55. T. Oakes, S. Powell, C. Castelnovo, A. Lamacraft, J. P. Garrahan, Phases of quantum dimers from ensembles of classical stochastic trajectories, Phys. Rev. B 98, 064302 (2018).

56. Z. Yan, Y. Wu, C. Liu, O. F. Syljuåsen, J. Lou, Y. Chen, Sweeping cluster algorithm for quantum spin systems with strong geometric restrictions, Phys. Rev. B 99, 165135 (2019).

57. J. Herzog-Arbeitman, S. Mantilla, I. Sodemann, Solving the quantum dimer and six vertex models one electric field line at a time, Phys. Rev. B 99, 245108 (2019).

58. R. K. Kaul, R. G. Melko, A. W. Sandvik, Bridging lattice-scale physics and continuum field theory with quantum Monte Carlo simulations, Annu. Rev. Con. Mat. Phys. 4, 179 (2013).

59. W. Bietenholz, J. C. Pinto Barros, S. Caspar, M. Hornung, U.-J. Wiese, Meron- and Semi-Vortex-Clusters as Physical Carriers of Topological Charge and Vorticity, PoS LATTICE2019 288, (2019).

60. H. Liu, S. Chandrasekharan, R. K. Kaul, Hamiltonian models of lattice fermions solvable by the meron-cluster algorithm, arXiv:2011.13208

61. D. Banerjee, F.-J. Jiang, T. Z. Olesen, P. Orland, U.-J. Wiese, From the SU(2) quantum link model on the honeycomb lattice to the quantum dimer model on the kagomé lattice: Phase transition and fractionalized flux strings, Phys. Rev. B 97, 20, 205108 (2018).

62. D. Banerjee, F.-J. Jiang, M. Kon, U.-J. Wiese, Real-Time Simulation of Large Open Quantum Spin Systems driven by Measurements, Phys. Rev. B 90, 241104 (2014).

63. F. Hebenstreit, D. Banerjee, M. Hornung, F.-J. Jiang, F. Schranz, U.-J. Wiese, Realtime dynamics of open quantum spin systems driven by dissipative processes, Phys. Rev. B 92, 035116 (2015).

64. D. Banerjee, F. Hebenstreit, F.-J. Jiang, U.-J. Wiese, Real-time simulation of nonequilibrium transport of magnetization in large open quantum spin systems driven by dissipation, Phys. Rev. B 92, 121104 (2015).

65. M. Lüscher, Peter Weisz, Locality and exponential error reduction in numerical lattice gauge theory, JHEP 0109, 010 (2001).

66. P. Majumdar, Experiences with the multilevel algorithm, Nucl. Phys. B Proc. Suppl. 119, 1021 (2003). 